\title{
Severity of associated weeds in Rabi crops and their control measures perceived by farmers
}

\author{
S. M. A. Islam ${ }^{1}$, M. A. I. Rana ${ }^{1}$, M. Ahmed ${ }^{2}$ and M. M. Islam² \\ Received: 23 ${ }^{\text {rd }}$ April 2020 / Accepted: $26^{\text {th }}$ July 2021
}

\section{ABSTRACT}

Purpose: The study was conducted to identify the associated weeds of Rabi (winter) crops and their control measures used by the farmers at Dumuria and Batiaghata upazila in Khulna district of Bangladesh.

Research Method: Data were collected from purposively selected 73 respondents using structured interview conducted from 4 January to 20 April, 2019.

Findings: In the study area, Echinochloa crussgalli and Cynodon dactylon were found as dominant within rice fields with the highest severity index of $25.34 \%$ and $20.54 \%$ respectively. In sesame fields, Cucumis melo var. agrestis and Cynodon dactylon were found with $16.78 \%$ and $12.33 \%$ severity index respectively. The severity index of Cynodon dactylon was the highest in majority of the vegetable crops. The weeding of rice, potato, bitter gourd, spinach, country bean was done within the critical weeding regime but in the rest of the crops weeding was not done during the critical weeding regime.

Originality/value: Farmers spend about $40 \%$ of the total production cost on weed control and the critical weeding regime has a major impact on effectiveness of weed control, overall economic benefit and net income.

Key Words: Associated weeds, Critical weeding regime, Rabi crops, Severity index

\section{INTRODUCTION}

Bangladesh is predominantly an agrarian country. The economy of Bangladesh is primarily dependent on agriculture. Agriculture contributes about $14 \%$ to the country's GDP. About $40 \%$ of the labor force is employed in agriculture (BBS, 2018). Due to very fertile land and favorable climatic conditions, a variety of crops are grown abundantly in the country during different seasons [Rabi (winter) and Kharif (summer)]. Amongst, Rabi season is important because of the increase in the income of farmers, as they grow many cereals, pulses, oilseeds and vegetables in this season.

Usually, crops are associated with different weeds. The weeds associated with most of the crops are crop-specific but not due to parasitic reasons. They may be associated with certain crops for specific microclimate, mimicry, and ready contamination of crop seeds (Gupta,
1998). Competition and Allelopathy are the important ecological interactions between the crop and weeds.

Crop weed competition has almost exclusively focused on the response of the crop, with only occasional reference to weed responses. Although crop yield loss is central to the shortterm goals of the farmer, longer term weed management goals, particularly those related to managing annual weeds, should consider weed seed yield and weed seed quality, both of which are affected by crop weed competition (Jordan, 1996; Norris, 2007).The weed competition in the crop field is invariably severe in the early stages of the crop than at later stages. Generally, in a crop of 100 days duration, the first 35 days after sowing should be maintained in a weed

\footnotetext{
1 Agrotechnology Discipline, Khulna University, Khulna-9208, Bangladesh

matiul_rubel@yahoo.com
}

https://orcid.org/0000-0001-7892-4666 
free condition. There is no need to attempt for a weed free condition throughout the life period of the crop, as it will entail unnecessary additional expenditure without a proportionate increase in yield (Walia and Walia, 2015). Thus, the field should be weed-free during the critical period of crop weed competition.

The critical period of weed crop competition is defined as that shortest period in the ontogeny of the crop when weeding will result in the highest economic returns (Gupta, 1998).The critical period of weed crop competition is the period from the time of sowing up to, in which the crop is to be maintained in a weed free environment to get the highest economical yield. In general, there is a hypothesis that for producing optimum yields, the annual crops require a weed free respite for the first one-fourth to onethird of their growing period. Longer duration weed control usually does not give additional economic return. Unfortunately, most farmers erroneously assume that removing weeds at any time during the growing season is good enough for obtaining full benefits of weeding in terms of increased crop yield (Gupta, 1998). In Bangladesh, weeds are traditionally controlled by hand weeding.

It has been estimated that losses in crop yields due to weeds in developed countries are 5\%, and in the least developed countries about $25 \%$ (Mukhopadhayay, 1992). The weeds reduce the crop yields, directly and indirectly, they elevate farm production costs through energy spent in controlling them. On country basis, such losses in crop yields have been estimated at $15-30 \%$ in wheat, $30-35 \%$ in rice, and $18-85 \%$ each in maize, sorghum, pulses, and oilseeds (Mukhopadhayay, 1992).

Considering the mentioned facts, this study was conducted to explore the knowledge on associated weeds and their management and to determine the socioeconomic condition of producers. Specifically, the objectives were to determine farmers' experience in weed identification and management. The data varied due to differences in knowledge, understanding, age, family status, level of education, and so on. These following specific objectives were formulated to achieve the research goals.

i.To identify major weeds associated with important Rabi crops as perceived by the farmers.

ii. To describe the socio-economic characteristics of the Rabi crop growers for depicting their competence in identifying the associated weeds.

iii. To find out the control measures of weeds practiced by the farmers.

iv.To explain the cost effectiveness of weed control methods.

\section{MATERIALS AND METHODS}

The study was conducted at few villages under three unions of each of Dumuria and Batiaghata Upazila of Khulna district. The farmers involved in Rabi crop cultivation were treated as the population of the area. The researchers purposively selected 73 farmers as respondents to conduct this survey (36 respondents from Batiaghata and 37 respondents from Dumuria). The respondents were from villages of Atlia, Bhandar Para, and Kharnia union of Dumuria Upazila and villages of Batiaghata, Jalma, and Gangarampur union of Batiaghata Upazila. Data were collected by face to face interviews with the farmers using the interview schedule conducted from 4 January to 20 April, 2019. The respondents were asked to mention the names of the weeds which they used to encounter in their Rabi season crop fields. The researchers made several field visits to observe the real crop field scenario sometimes with the farmers, and sometimes alone themselves to cross check the authenticity of the information provided by farmers. Safeguarding the obtained data was maintained this way and a total of 18 associated weeds were identified this way. The Associated Weed Severity Index Score (AWSIS) was determined by following the very common formula:

AWSIS $=\mathrm{N}_{1} \times 4+\mathrm{N}_{2} \times 3+\mathrm{N}_{3} \times 2+\mathrm{N}_{4} \times 1+\mathrm{N}_{5} \times 0$

Where, 
$\mathrm{N}_{1}=$ Number of the respondent identified the weed and rated as very severe

$\mathrm{N}_{2}=$ Number of the respondent identified the weed and rated as severe

$\mathrm{N}_{3}=$ Number of the respondent identified the weed and rated as moderately severe

$\mathrm{N}_{4}=$ Number of the respondent identified the weed and rated as less severe

$\mathrm{N}_{5}=$ Number of the respondent identified the weed and rated as not severe

After determination of the associated weed severity index score associated weed severity index was determined by the following formula:

(\%)Severity of the weed $=$

$\frac{\text { (Observed associated weed severity index score) }}{\text { (Possible highest associated weed severity index score) }} \times 100$

As there were 73 respondents associated, weed severity index score could range 0-292 where ' 0 ' indicates no association of weed and ' 292 ' indicates the highest weed association. The SPSS software was used for data analysis [for measuring the mean, frequency, percentage, standard deviation (SD), maximum, minimum, data categorization, correlation coefficient calculation, etc.].

The researchers felt that the information providers' overall socioeconomic as well as agricultural competencies must be assessed. In this context, nine socioeconomic characteristics (considered as variables) of the respondents were used in the study: age, educational qualifications, family size, farming experience, farm size, cosmopolitanism, organizational participation, extension media contact, and agricultural training. Descriptive statistics like the number, percentage, range, mean and standard deviation were used in describing selected variables of the study. Practiced weed control measures and most preferred weed control measures were also explored as per the opinion of the farmers.
Critical weeding regimes for the identified associated weeds were established based on the information collected from Hand Book of Agricultural Technology (Chowdhury and Hassan, 2013), and panel review, expert opinion, etc. Pencil sketches were drawn to describe the utility of the weeding within the critical weeding regimes to ensure maximum effectiveness along with the economic benefit. An economic analysis was also performed to depict the possibility of profit maximization through weeding cost reduction.

\section{RESULTS AND DISCUSSION}

\section{Identification of associated weeds of Rabi crops and their control measure as perceived by the farmers}

Batiaghata and Dumuria Upazila of Khulna districts are famous for growing various vegetables. The farmers, selected for this study, used to grow various crops like rice, sesame, mungbean, mustard, tomato, potato, pumpkin, bitter gourd, cauliflower, cabbage, Indian spinach, yard long bean, okra, brinjal, spinach, country bean, etc. (Table 01). They grew all these crops in the survey year or the year before the survey. According to interviews with the farmers about the associated weed of Rabi crops in Dumuria and Batiaghata Upazila it was found that several weeds were associated with the crops at that time. Weeds belonging to different families were found associated with these crops are discussed with their severity index in Table 01 . A total of 18 associated weeds were identified in this way (Table 01).

Among the associated weeds with rice Echinochloa crussgalli was found with the highest severity index (25.34\%) which ranked the $1^{\text {st }}$ followed by Cynodon dactylon with severity index $20.54 \%$ which ranked $2^{\text {nd }}$ and Cyperus rotundus ranked $3^{\text {rd }}$ with a severity index of $15.05 \%$. Paspalum distichum and Alternanthera philoxeroides were with a $14.72 \%$ severity index. Oxalis europaea, Azolla pinnata, Distichlis spicata, Enhydra fluctuans with a severity index of $10.61 \%, 6.14 \%, 3.42 \%$, and $1.71 \%$ respectively. Weeds associated with 
Table 01. Intensity and rank position of different weed associated with Rabi crops (rice, mungbean, grass pea, sesame, mustard, tomato, potato and country bean).

\begin{tabular}{|c|c|c|c|c|c|c|c|c|c|}
\hline \multirow[t]{2}{*}{ Serial } & \multirow{2}{*}{$\begin{array}{l}\text { Name of the weed } \\
\text { (Local name) English name }\end{array}$} & \multicolumn{8}{|c|}{ Crops } \\
\hline & & Rice & Mungbean & Grass pea & Sesame & Mustard & Tomato & Potato & $\begin{array}{c}\text { Country } \\
\text { bean }\end{array}$ \\
\hline 1 & $\begin{array}{l}\text { Cynodon dactylon } \\
\text { (Durba)Bermuda grass }\end{array}$ & $\begin{array}{c}2^{\text {nd }} \\
(20.54 \%)\end{array}$ & $\begin{array}{c}2^{\text {nd }} \\
(9.93 \%)\end{array}$ & $\begin{array}{c}1^{\mathrm{st}} \\
(4.10 \%)\end{array}$ & $\begin{array}{c}2^{\text {nd }} \\
(12.33 \%)\end{array}$ & $\begin{array}{c}2^{\text {nd }} \\
(1.37 \%)\end{array}$ & $\begin{array}{c}1^{\mathrm{st}} \\
(5.48 \%)\end{array}$ & $\begin{array}{c}1^{\mathrm{st}} \\
(2.39 \%)\end{array}$ & $\begin{array}{c}1^{\text {st }} \\
(3.08 \%)\end{array}$ \\
\hline 2. & $\begin{array}{l}\text { Echinochloa crussgalli } \\
\text { (Shama)Barnyard grass }\end{array}$ & $\begin{array}{c}1^{\mathrm{st}} \\
(25.34 \%)\end{array}$ & $\begin{array}{c}4^{\text {th }} \\
(3.08 \%)\end{array}$ & $\begin{array}{c}2^{\text {nd }} \\
(3.08 \%)\end{array}$ & $\begin{array}{c}4^{\text {th }} \\
(4.45 \%)\end{array}$ & $\begin{array}{c}4^{\text {th }} \\
(0.68 \%)\end{array}$ & $\begin{array}{c}4^{\text {th }} \\
(1.36 \%)\end{array}$ & & \\
\hline 3. & $\begin{array}{l}\text { Cyperus rotundus } \\
\text { (Mutha)Com. Nut Sedge }\end{array}$ & $\begin{array}{c}3^{\text {rd }} \\
(15.05 \%)\end{array}$ & & & & $\begin{array}{c}3^{\text {rd }} \\
(1.02 \%)\end{array}$ & $\begin{array}{c}3^{\text {rd }} \\
(1.76 \%)\end{array}$ & $\begin{array}{c}3^{\text {rd }} \\
(0.68 \%)\end{array}$ & $\begin{array}{c}3^{\text {rd }} \\
(2.05 \%)\end{array}$ \\
\hline 4. & $\begin{array}{l}\text { Paspalum distichum } \\
\text { (Gitlaghash)Knotgrass }\end{array}$ & $\begin{array}{c}4^{\text {th }} \\
(14.72 \%)\end{array}$ & $\begin{array}{c}3^{\text {rd }} \\
(8.9 \%)\end{array}$ & & $\begin{array}{c}3^{\text {rd }} \\
(9.24 \%)\end{array}$ & $\begin{array}{c}1^{\mathrm{st}} \\
(2.05 \%)\end{array}$ & $\begin{array}{c}2^{\text {nd }} \\
(2.05 \%)\end{array}$ & $\begin{array}{c}4^{\text {th }} \\
(0.34 \%)\end{array}$ & $\begin{array}{c}2^{\text {nd }} \\
(2.74 \%)\end{array}$ \\
\hline 5. & $\begin{array}{l}\text { Cucumis melo var. agrestis } \\
\text { (Bunobangi)Wild melon }\end{array}$ & & $\begin{array}{c}1^{\mathrm{st}} \\
(14.72 \%)\end{array}$ & & $\begin{array}{c}1^{\mathrm{st}} \\
(16.78 \%)\end{array}$ & & & & $\begin{array}{c}4^{\text {th }} \\
(1.02 \%)\end{array}$ \\
\hline 6. & $\begin{array}{l}\text { Corchorus trilocularis } \\
\text { (Bunokosta)Wild Jute }\end{array}$ & & $\begin{array}{c}5^{\text {th }} \\
(2.73 \%)\end{array}$ & & $\begin{array}{l}5^{\text {th }} \\
(2.73 \%)\end{array}$ & & & & $\begin{array}{c}4^{\text {th }} \\
(1.02 \%)\end{array}$ \\
\hline 7. & $\begin{array}{l}\text { Physalis heterophylla } \\
\text { (Foska begun)Clammy ground } \\
\text { cherry }\end{array}$ & & $\begin{array}{c}6^{\text {th }} \\
(1.37 \%)\end{array}$ & & & & $\begin{array}{c}6^{\text {th }} \\
(0.68 \%)\end{array}$ & & \\
\hline 8. & $\begin{array}{l}\text { Xanthium indicum } \\
\text { (Ghagra)Rough cocklebur }\end{array}$ & & $\begin{array}{c}7^{\text {th }} \\
(1.02 \%)\end{array}$ & & $\begin{array}{c}6^{\text {th }} \\
(2.40 \%)\end{array}$ & & & & \\
\hline 9. & $\begin{array}{l}\text { Alternanthera philoxeroides } \\
\text { (Malancha)Alligator weed }\end{array}$ & $\begin{array}{c}4^{\text {th }} \\
(14.72 \%)\end{array}$ & & $\begin{array}{c}3^{\text {rd }} \\
(2.73 \%)\end{array}$ & & & & & \\
\hline 10. & $\begin{array}{l}\text { Enhydra fluctuans } \\
\text { (Helencha)Buffalo } \\
\text { spinach }\end{array}$ & $\begin{array}{c}8^{\text {th }} \\
(1.71 \%)\end{array}$ & & & & & & & \\
\hline 11. & $\begin{array}{l}\text { Amaranthus viridis } \\
\text { (Shaknotey)Slender amaranth }\end{array}$ & $\begin{array}{c}9^{\text {th }} \\
(1.08 \%)\end{array}$ & & & & & $\begin{array}{c}5^{\text {th }} \\
(1.02 \%)\end{array}$ & $\begin{array}{c}2^{\text {nd }} \\
(1.02 \%)\end{array}$ & \\
\hline 12. & $\begin{array}{l}\text { Oxalis europaea } \\
\text { (Amrulshak)Yellow } \\
\text { woodsorrel }\end{array}$ & $\begin{array}{c}5^{\text {th }} \\
(10.61 \%)\end{array}$ & & & & & & & \\
\hline 13 & $\begin{array}{l}\text { Azolla pinnata } \\
\text { (Shawla)Mosquito fern }\end{array}$ & $\begin{array}{c}6^{\text {th }} \\
(6.14 \%)\end{array}$ & & & & & & & \\
\hline 14. & $\begin{array}{l}\text { Distichlis spicata } \\
\text { (Nunta)Seashore saltgrass }\end{array}$ & $\begin{array}{c}7^{\text {th }} \\
(3.42 \%)\end{array}$ & & & & & & & $\begin{array}{c}6^{\text {th }} \\
(0.34 \%)\end{array}$ \\
\hline 15. & $\begin{array}{l}\text { Croton sparsiflorus } \\
\text { (Bonmorich)Rushfoil }\end{array}$ & & & & $\begin{array}{c}7^{\text {th }} \\
(2.05 \%)\end{array}$ & & & & \\
\hline 16. & $\begin{array}{l}\text { Eleusine indica } \\
\text { (Chapra)Indian goosegrass }\end{array}$ & & & & & & & $\begin{array}{c}2^{\text {nd }} \\
(1.02 \%)\end{array}$ & $\begin{array}{c}5^{\text {th }} \\
(0.68 \%)\end{array}$ \\
\hline 17. & $\begin{array}{l}\text { Chenopodium album } \\
\text { (Bothua)Lamb's quarters }\end{array}$ & & & & & & & & \\
\hline 18. & $\begin{array}{l}\text { Raphanus raphanistrum } \\
\text { (Bonmula)Wild radish }\end{array}$ & & & & & & & & \\
\hline
\end{tabular}


Table 01. Continued... (rice, mungbean, grass pea, sesame, mustard, tomato, potato and country bean).

\begin{tabular}{|c|c|c|c|c|c|c|c|c|c|c|}
\hline \multirow[t]{2}{*}{ Serial } & \multirow{2}{*}{$\begin{array}{c}\text { Name of the weed } \\
\text { (Local name) English name }\end{array}$} & \multicolumn{8}{|c|}{ Crops } & \multirow[b]{2}{*}{ Spinach } \\
\hline & & Okra & $\begin{array}{l}\text { Indian } \\
\text { spinach }\end{array}$ & $\begin{array}{l}\text { Bitter } \\
\text { gourd }\end{array}$ & $\begin{array}{l}\text { Yard } \\
\text { long } \\
\text { bean }\end{array}$ & Pumpkin & Cabbage & Cauliflower & Brinjal & \\
\hline 1 & $\begin{array}{l}\text { Cynodon dactylon } \\
\text { (Durba)Bermuda grass }\end{array}$ & $\begin{array}{c}1^{\text {st }} \\
(3.08 \%)\end{array}$ & $\begin{array}{c}1^{\text {st }} \\
(1.36 \%)\end{array}$ & $\begin{array}{c}1^{\text {st }} \\
(5.13 \%)\end{array}$ & $\begin{array}{c}1^{\mathrm{st}} \\
(5.14 \%)\end{array}$ & $\begin{array}{c}1^{\text {st }} \\
(2.73 \%)\end{array}$ & $\begin{array}{c}1^{\mathrm{st}} \\
(1.37 \%)\end{array}$ & $\begin{array}{c}1^{\mathrm{st}} \\
(7.53 \%)\end{array}$ & $\begin{array}{c}1^{\text {st }} \\
(3.77 \%)\end{array}$ & $\begin{array}{c}1^{\text {st }} \\
(2.05 \%)\end{array}$ \\
\hline 2. & $\begin{array}{l}\text { Echinochloa crussgalli } \\
\text { (Shama)Barnyard grass }\end{array}$ & & & $\begin{array}{c}2^{\text {nd }} \\
(2.74 \%)\end{array}$ & & & & $\begin{array}{c}3^{\text {rd }} \\
(2.05 \%)\end{array}$ & & \\
\hline 3. & $\begin{array}{l}\text { Cyperus rotundus } \\
\text { (Mutha)Com. Nut Sedge }\end{array}$ & $\begin{array}{c}3^{\text {rd }} \\
(1.02 \%)\end{array}$ & $\begin{array}{c}1^{\mathrm{st}} \\
(1.36 \%)\end{array}$ & & $\begin{array}{c}2^{\text {nd }} \\
(4.10 \%)\end{array}$ & & $\begin{array}{c}2^{\text {nd }} \\
(1.03 \%)\end{array}$ & $\begin{array}{c}2^{\text {nd }} \\
(6.85 \%)\end{array}$ & & $\begin{array}{c}1^{\mathrm{st}} \\
(2.05 \%)\end{array}$ \\
\hline 4. & $\begin{array}{l}\text { Paspalum distichum } \\
\text { (Gitlaghash)Knotgrass }\end{array}$ & $\begin{array}{c}2^{\text {nd }} \\
(2.05 \%)\end{array}$ & & $\begin{array}{c}5^{\text {th }} \\
(1.71 \%)\end{array}$ & $\begin{array}{c}3^{\text {rd }} \\
(2.05 \%)\end{array}$ & & $\begin{array}{c}2^{\text {nd }} \\
(1.03 \%)\end{array}$ & & $\begin{array}{c}3^{\text {rd }} \\
(1.71 \%)\end{array}$ & $\begin{array}{c}3^{\text {rd }} \\
(0.34 \%)\end{array}$ \\
\hline 5. & $\begin{array}{l}\text { Cucumis melo var. agrestis } \\
\text { (Bunobangi)Wild melon }\end{array}$ & $\begin{array}{c}1^{\mathrm{st}} \\
(3.08 \%)\end{array}$ & & $\begin{array}{c}3^{\text {rd }} \\
(2.42 \%)\end{array}$ & & $\begin{array}{c}2^{\text {nd }} \\
(1.71 \%)\end{array}$ & & & & \\
\hline 6. & $\begin{array}{l}\text { Corchorus trilocularis } \\
\text { (Bunokosta)Wild Jute }\end{array}$ & $\begin{array}{c}3^{\text {rd }} \\
(1.02 \%)\end{array}$ & & $\begin{array}{c}4^{\text {th }} \\
(2.05 \%)\end{array}$ & & $\begin{array}{c}2^{\text {nd }} \\
(1.71 \%)\end{array}$ & & & & \\
\hline 7. & $\begin{array}{l}\text { Physalis heterophylla } \\
\text { (Foska begun)Clammy } \\
\text { ground cherry }\end{array}$ & & & & & & & & & \\
\hline 8. & $\begin{array}{l}\text { Xanthium indicum } \\
\text { (Ghagra)Rough cocklebur }\end{array}$ & & & & & & & & & \\
\hline 9. & $\begin{array}{l}\text { Alternanthera philoxeroides } \\
\text { (Malancha)Alligator weed }\end{array}$ & & & & & & & & & \\
\hline 10. & $\begin{array}{l}\text { Enhydra fluctuans } \\
\text { (Helencha)Buffalo } \\
\text { spinach }\end{array}$ & & & & & & & & & \\
\hline 11. & $\begin{array}{l}\text { Amaranthus viridis } \\
\text { (Shaknotey)Slender amaranth }\end{array}$ & & & & & $\begin{array}{c}3^{\text {rd }} \\
(0.68 \%)\end{array}$ & $\begin{array}{c}3^{\text {rd }} \\
(0.68 \%)\end{array}$ & $\begin{array}{c}4^{\text {th }} \\
(1.71 \%)\end{array}$ & & \\
\hline 12. & $\begin{array}{l}\text { Oxalis europaea } \\
\text { (Amrulshak)Yellow } \\
\text { woodsorrel }\end{array}$ & & & & & & & & & \\
\hline 13 & $\begin{array}{l}\text { Azolla pinnata } \\
\text { (Shawla)Mosquito fern }\end{array}$ & & & & & & & & & \\
\hline 14. & $\begin{array}{l}\text { Distichlis spicata } \\
\text { (Nunta)Seashore saltgrass }\end{array}$ & & & & & & & & $\begin{array}{c}4^{\text {th }} \\
(1.02 \%)\end{array}$ & \\
\hline 15. & $\begin{array}{l}\text { Croton sparsiflorus } \\
\text { (Bonmorich)Rushfoil }\end{array}$ & & & & & & & & & \\
\hline 16. & $\begin{array}{l}\text { Eleusine indica } \\
\text { (Chapra)Indian goosegrass }\end{array}$ & & & & $\begin{array}{c}4^{\text {th }} \\
(1.02 \%)\end{array}$ & & & & & \\
\hline 17. & $\begin{array}{l}\text { Chenopodium album } \\
\text { (Bothua)Lamb's quarters }\end{array}$ & & & $\begin{array}{c}6^{\text {th }} \\
(1.02 \%)\end{array}$ & & & & $\begin{array}{c}5^{\text {th }} \\
(1.37 \%)\end{array}$ & $\begin{array}{c}4^{\text {th }} \\
(1.02 \%)\end{array}$ & \\
\hline 18. & $\begin{array}{l}\text { Raphanus raphanistrum } \\
\text { (Bonmula)Wild radish }\end{array}$ & & & & & & & $\begin{array}{c}5^{\text {th }} \\
(1.37 \%)\end{array}$ & $\begin{array}{c}2^{\text {nd }} \\
(3.76 \%)\end{array}$ & $\begin{array}{c}2^{\text {nd }} \\
(1.02 \%)\end{array}$ \\
\hline
\end{tabular}


mungbean were Cucumis melo var. agrestis with the highest severity index (14.72\%) ranked $1^{\text {st }}$ followed by Cynodon dactylon with the $2^{\text {nd }}$ highest severity index of $9.93 \%$ and Paspalum distichum with the $3^{\text {rd }}$ highest severity index of $8.9 \%$, Echinochloa crussgalli, Corchorus trilocularis, and Physalis heterophylla with a severity index of $3.08 \%, 2.73 \%, 1.37 \%$ respectively. Associated weeds with grass pea were Cynodon dactylon with the highest severity index $(4.10 \%)$ which ranked $1^{\text {st }}$ followed by Echinochloa crussgalli with the $2^{\text {nd }}$ highest severity index (3.08\%). Associated weeds with sesame were Cucumis melo var. agrestis with the highest severity index of $16.78 \%$ and ranked $1^{\text {st }}$ followed by Cynodon dactylon with the $2^{\text {nd }}$ highest severity index (12.33\%) and Paspalum distichum ranked $3^{\text {rd }}$ with a $9.24 \%$ severity index. Echinochloa crussgalli, Corchorus trilocularis, Xanthium indicum, Croton sparsiflorus were also associated with a severity index of $4.45 \%$, $2.73 \%, 2.4 \%$, and $1.02 \%$ respectively. Among the associated weed with mustard, Paspalum distichum was found with the highest severity index $(2.05 \%)$ which ranked $1^{\text {st }}$ followed by Cynodon dactylon with the $2^{\text {nd }}$ highest severity index $(1.37 \%)$ and Cyperus rotundus with the $3^{\text {rd }}$ highest severity index $(1.02 \%)$. During Rabi season Cynodon dactylon was found associated with the majority of vegetable crops such as tomato, potato, bean, Indian spinach, bitter gourd, yard long bean, pumpkin, cabbage, cauliflower, spinach with the highest severity index. Other weeds associated with vegetables were Paspalum distichum, Eleusine indica, Cyperus rotundus, Amaranthus viridis, Corchorus trilocularis, Cucumis melo var. agrestis, Chenopodium album, Echinochloa crussgalli, Raphanus raphanistrum.

Huda et al., (2017) conducted a weed survey in the Agronomy Field Laboratory of BAU (Bangladesh Agricultural University) Farm and farmers' fields of Sutiakhali Natunchar village in Mymensingh district. In both rice fields of the Agronomy Field Laboratory of BAU Farm Eleocharisatro purpurea had the highest abundance value but in farmers' field Cyperus difformis occupied the highest value and other dominant weed species were Leersia hexandra, Monochoria vaginalis, Echinochloa crusgalli,
Paspalum distichum, and Alternanthera philoxeroides.

Islam et al., (2018) experimented the Agronomy Field Laboratory of BAU, Mymensingh to study the effect of different weed management techniques on the performance of wheat cultivars and found Polygonum orientale, Chenopodium album, Cynodon dactylon, Sonchu sarvensis and Cyperus rotundus as the dominant weed species and application of rice straw mulch technique appears to be the promising technology in controlling weeds. Dangwal et al., (2010) conducted a survey in Nowshera teshil of the district Rajouri (Jammu \& Kashmir) of India, and they found Avena fatua, Anagalli sarvensis, Chenopodium album, Cirsium arvense, Fumaria parviflora, Lathyrus aphaca, Melilotus indica, Parthenium hysterophorus, Phalaris minor, Rumex dentatus, Vicia hirsuta and Vicia sativa, etc. as the common weeds of Rabi crops.

Comparison between the prescribed weeding regime and practiced weeding regime along with the cost and method of different Rabi crops was done. The study was conducted to study associated weed with a particular crop, weeding time, weeding frequency, and cost of weeding and method of weeding practiced by the farmers (Table 02). The weeding frequency of the seventeen studied crops ranged from one to three. Most of the crops (rice, mungbean, sesame, mustard, potato, country bean, okra, Indian spinach, bitter gourd, pumpkin) were weeded twice. At vegetable crops (tomato, yard long bean, cabbage, cauliflower, and brinjal) three sessions of weeding were practiced by farmers. Only one weeding was given in grass pea and spinach. The average weeding cost of rice cabbage, cauliflower, and brinjal was maximum $\left(10,000\right.$ BDT ha $\left.{ }^{-1}\right)$. Tomato was weeded thrice and weeding cost was 9,600 BDT $\mathrm{ha}^{-1}$. Average weeding cost of mungbean $(8,800$ BDT ha-1) sesame and okra $\left(8,000\right.$ BDT ha $\left.^{-1}\right)$, followed by potato and country bean $(6,400$ BDT ha ${ }^{-1}$ ) and mustard, bitter gourd and yard long bean $\left(6,000\right.$ BDT ha $\left.^{-1}\right)$. Average weeding was found lesser in Indian spinach $(4,800 \mathrm{BDT}$ $\left.\mathrm{ha}^{-1}\right)$ and pumpkin $\left(4,000 \mathrm{BDTha}^{-1}\right)$. The least cost of weeding was observed in spinach $(2,000$ BDT ha $^{-1}$ ) (Table 02) 
Table 02. Comparison between prescribed weeding regime and practiced weeding regime along with cost and method for different Rabi crops.

\begin{tabular}{|c|c|c|c|c|c|c|}
\hline Crop Name & $\begin{array}{c}\text { Avg. } \\
\text { no of } \\
\text { observed } \\
\text { weeding }\end{array}$ & $\begin{array}{l}\text { Suitable } \\
\text { weeding } \\
\text { regime* }\end{array}$ & $\begin{array}{c}\text { Observed } \\
\text { weeding DAS/ } \\
\text { DAT }\end{array}$ & $\begin{array}{c}\text { Average } \\
\text { weeding cost } \\
\text { ha-1 (BDT) }\end{array}$ & $\begin{array}{l}\text { Usual method } \\
\text { of weeding }\end{array}$ & $\begin{array}{c}\text { Cost effective } \\
\text { method }\end{array}$ \\
\hline Rice & 2 & 20-45 DAT & $\begin{array}{c}30 \text { DAT, } 45 \\
\text { DAT }\end{array}$ & 10,000 & Physical & Chemical \\
\hline Mungbean & 2 & 25-40 DAS & $\begin{array}{l}10 \text { DAS, } 30 \\
\text { DAS }\end{array}$ & 8,800 & Physical & Chemical \\
\hline Grass pea & 1 & 25-40 DAS & $15 \mathrm{DAS}$ & 2,200 & Physical & Chemical \\
\hline Sesame & 2 & 15-45 DAS & $\begin{array}{l}15 \text { DAS, } 25 \\
\text { DAS }\end{array}$ & 8,000 & Physical & Chemical \\
\hline Mustard & 2 & 20-40 DAS & $\begin{array}{l}10 \text { DAS, } 30 \\
\text { DAS }\end{array}$ & 6,000 & Physical & Chemical \\
\hline Tomato & 3 & 30-45 DAT & $\begin{array}{c}10 \text { DAT, } 30 \\
\text { DAT, } 45 \text { DAT }\end{array}$ & 9,600 & Physical & Chemical \\
\hline Potato & 2 & 20-40 DAS & $\begin{array}{l}20 \text { DAS, } 35 \\
\text { DAS }\end{array}$ & 6,400 & Physical & Chemical \\
\hline Country bean & 2 & 20-30 DAS & $\begin{array}{c}15 \text { DAS, } 30 \\
\text { DAS }\end{array}$ & 6,400 & Physical & Chemical \\
\hline Okra & 2 & 15-30 DAS & $\begin{array}{l}30 \text { DAS, } 45 \\
\text { DAS }\end{array}$ & 8,000 & Physical & Chemical \\
\hline Indian spinach & 2 & 20-30 DAT & $\begin{array}{c}20 \text { DAS, } 35 \\
\text { DAS }\end{array}$ & 4,800 & Physical & Chemical \\
\hline Bitter gourd & 2 & 30-45 DAS & $\begin{array}{l}30 \text { DAS, } 45 \\
\text { DAS }\end{array}$ & 6,000 & Physical & Chemical \\
\hline $\begin{array}{l}\text { Yard long } \\
\text { bean }\end{array}$ & 3 & 15-30 DAS & $\begin{array}{c}25 \mathrm{DAS}, 30 \\
\mathrm{DAS}, 50 \mathrm{DAS}\end{array}$ & 6,000 & Physical & Chemical \\
\hline Pumpkin & 2 & 20-30 DAS & $\begin{array}{l}30 \text { DAS , } 40 \\
\text { DAS }\end{array}$ & 4,000 & Physical & Chemical \\
\hline Cabbage & 3 & 30-45 DAS & $\begin{array}{c}7 \text { DAS, } 20 \text { DAS, } \\
35 \text { DAS }\end{array}$ & 10,000 & Physical & Chemical \\
\hline Cauliflower & 3 & 30-45 DAS & $\begin{array}{c}7 \text { DAS, } 20 \text { DAS, } \\
35 \text { DAS }\end{array}$ & 10,000 & Physical & Chemical \\
\hline Brinjal & 3 & 20-40 DAS & $\begin{array}{c}7 \text { DAT, } 20 \text { DAT, } \\
\text { 35 DAT }\end{array}$ & 10,000 & Physical & Chemical \\
\hline Spinach & 1 & $10-25 \mathrm{DAS}$ & $20 \mathrm{DAS}$ & 2,000 & Physical & Chemical \\
\hline
\end{tabular}

*Information was collected from Hand Book of Agricultural Technology, http://agritech.tnau.ac.in/agriculture/ agri_weemgt_criticalperiodofweedcompetition.html (Retrieved on 30 October, 2019), and from panel review, expert opinion, etc.

In this study, it was found that farmers do not have enough knowledge about a suitable weeding time, sometimes they conduct more unnecessary weeding before or after the critical weeding regime. For that reason, they have to spend extra money which increases the production cost and ultimately reduces the income from that particular crop.
Acharya and Bhattacharya (2013) reported that transplanted boro rice 20 to 45 days after transplanting (DAT) is the most critical period of weed crop competition. Singh et al., (1971) found that in cotton, good weed control was necessary for up to two weeks after crop emergence. Delay in weeding caused unrecoverable losses in the lint yield. Groundnut, 
a slow prostrate growing crop, required freedom from weeds almost up to 6-8 weeks after emergence to obtain optimum yields (Rethinam et al., 1976).

In sugarcane Singh et al., (1980) found 4 to 16 weeks period after planting is critical for the competition from weeds. In potato, only 2 to 4 weeks period after planting was considered as crucial for weeding. Delayed weeding caused considerable shrinkage in tuber yield (Gupta et al., 1979). A rapidly growing crop like mungbean responded most to weeding 35 days after sowing (Singh, 1978). Considering the importance of critical weed control period for increased crop production, the Ministry of Agriculture, Food and Rural Affairs of Canada regulary publishes critical weed-free period for their major agricultural crops (Ontario Crop IPM, 2020). Knezevic et al. (2002) reported that the critical period for weed control (CPWC) must be controlled to prevent yield losses. Knowing the CPWC is useful in making decisions on the need for and timing of weed control and in achieving efficient herbicide use from both biological and economic perspectives.

Weeding regime with a sketch-pictorial view was also studied in this research (Fig. 1, 2, 3, 4 and 5). In boro season rice, suitable weeding time ranges between 25 to 45 DAT and farmers' do weeding at 30 DAT and 45 DAT (Fig. 1). In sesame, suitable weeding time ranges between 15 to 45 DAS and farmers' practice weeding at 15 DAS and 25 DAS (Fig. 2). In mungbean, suitable weeding time range is between 25 to 40 DAS, and farmers' do weeding at 10 DAS and 30 DAS (Fig. 3). In potato, suitable weeding time range is between 20 to 40 DAS and farmers' practice weeding at 20 DAS and 35 DAS (Fig. 4). In tomato, suitable weeding time range is between 30 to 45 DAT and farmers' do weeding at 10 DAT, 30 DAT, and 45 DAT (Fig. 5 ). Weeding is done within the suitable weeding time in rice, sesame, and potato which is effective for weed control. In the case of mungbean and tomato, weeding is not done within a suitable weeding time which is ineffective and results in the increasing of weeding cost.
Socio-economic characteristics of the Rabi crop growers for depicting their competence in identifying the associated weeds

The researchers felt that the associated weed identification must be reliable although they were identified as per the perception provided by the farmers on good faith. All the information regarding the associated weeds, severity, control measures along with costs and benefits were collected from the farmers through the face-to-face survey. Thus the researchers felt that the information providers' overall socioeconomic as well as agricultural competencies must be assessed. It was found that the highest proportion (38.4\%) of the respondents belonged to the middle age category followed by $34.2 \%$ old aged and $27.4 \%$ young aged (Table 03 ). The highest portion $(41.1 \%)$ of the respondents had a secondary level of education while $23.3 \%$ could sign only, $15.1 \%$ had primary level of education, $8.2 \%$ had higher secondary level of education, $6.8 \%$ were illiterate, $2.7 \%$ had bachelor degree and $2.7 \%$ had the post-graduate level of education. The majority (53.4\%) of the respondents had small sized families while $26 \%$ of the respondents had medium sized families and $20.5 \%$ of the respondents had large sized families. About half (50.7\%) of the respondents had a high farming experience while $35.6 \%$ of the respondents had a medium farming experience and $13.7 \%$ of the respondents had a low farming experience. Majority of the respondents $(53.4 \%)$ had a small sized farm while $26 \%$ of the respondents had a medium sized farm and only $20.5 \%$ of the respondents had a large farm. Cosmopolitanism status was low to the medium category and had the same percentage (49.3\%), and only $1.4 \%$ was highly cosmopolite. Most $(84.9 \%)$ of respondents had a low organizational participation. More than half $(57.5 \%)$ of the respondents had a medium extension media contact while $39.7 \%$ had a low extension contact exposure and only $2.7 \%$ had a high extension media contact (Table 03 ).

Among the 73 respondents, 27 respondents were facilitated with agricultural training, and rest 46 respondents do not have an agricultural training. Different training providing organizations such as Department of Agricultural Extension(DAE), 


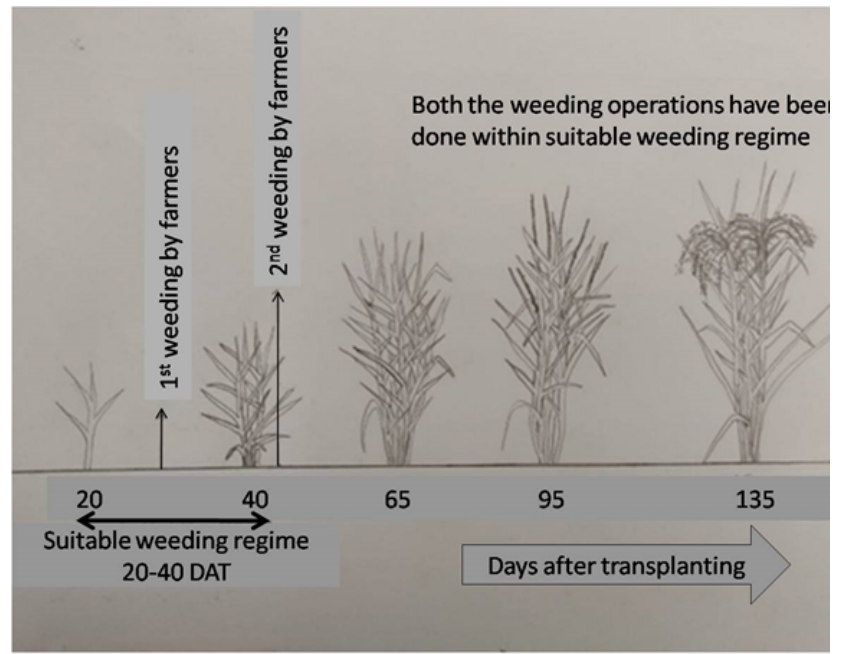

Figure 01. Weeding regime of rice.
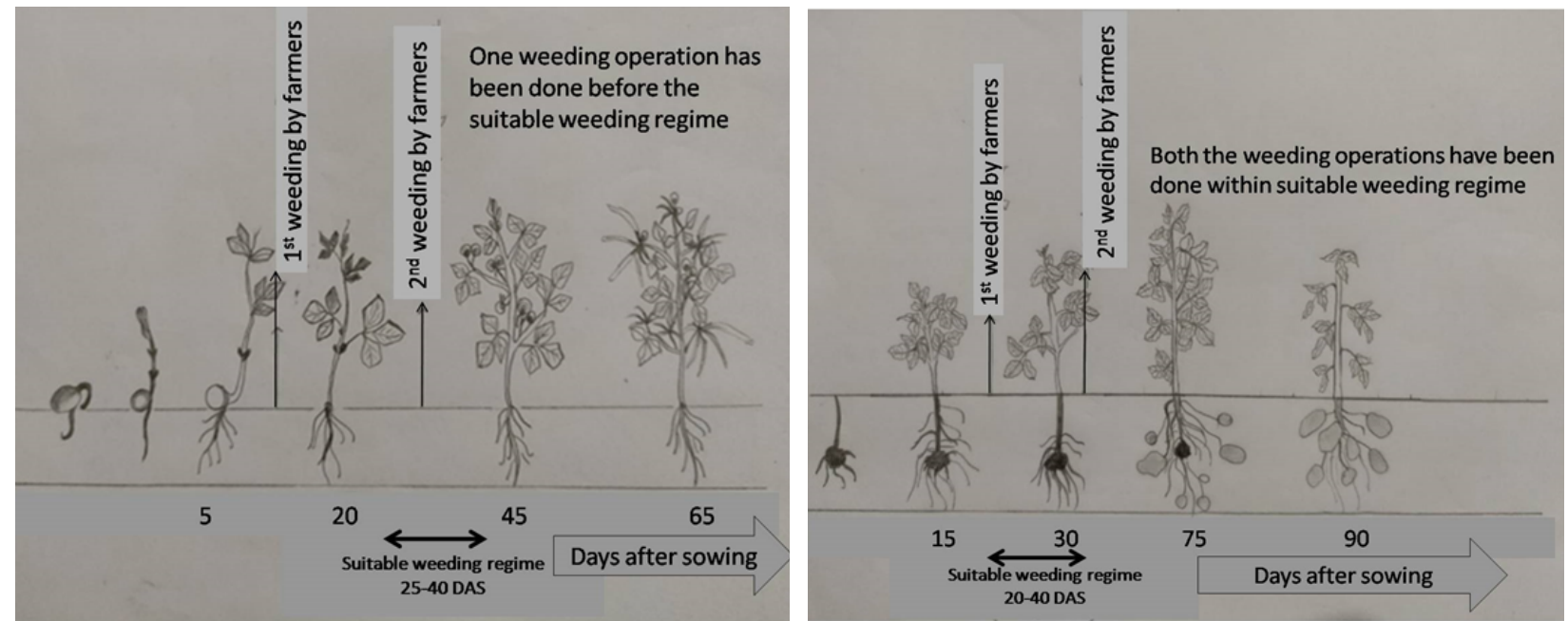

Figure 03. Weeding regime of mungbean. Figure 04. Weeding regime of potato.

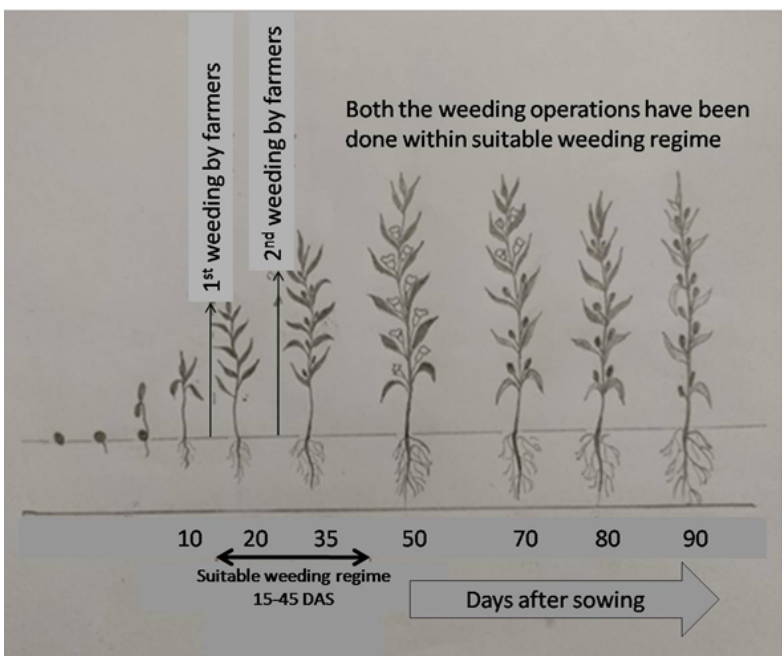

Figure 02. Weeding regime of sesame.

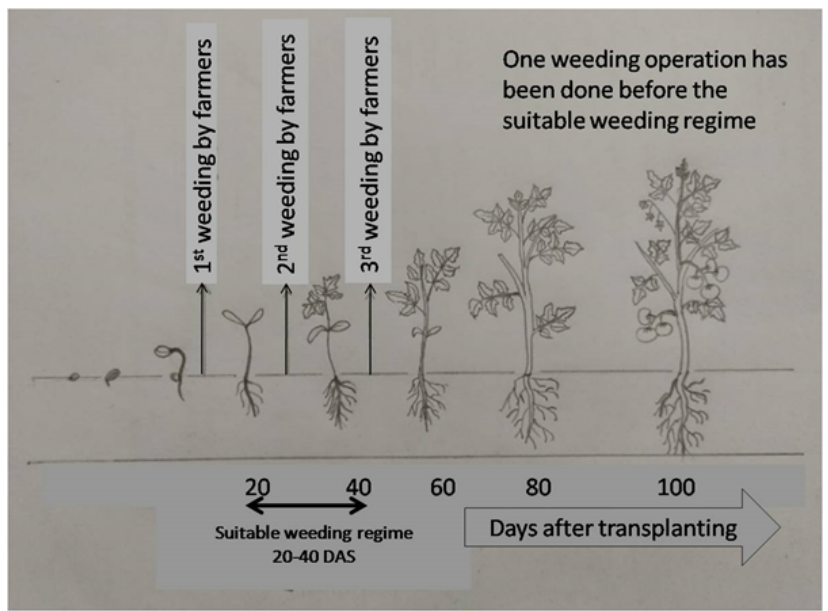

Figure 05. Weeding regime of tomato. 
Table 03. Distribution of the respondents on the basis of selected characteristics.

\begin{tabular}{|c|c|c|c|c|c|c|c|c|}
\hline \multirow[t]{2}{*}{ Characteristics } & \multirow[t]{2}{*}{ Categories } & \multirow[t]{2}{*}{ Scores } & \multicolumn{2}{|c|}{ Respondents $(\mathrm{N}=73)$} & \multirow[t]{2}{*}{ Mean } & \multirow[t]{2}{*}{ SD } & \multirow[t]{2}{*}{ Min. } & \multirow[t]{2}{*}{ Max. } \\
\hline & & & Frequency & Percentage & & & & \\
\hline \multirow{3}{*}{$\begin{array}{l}\text { Age } \\
\text { (Years) }\end{array}$} & Young & $\leq 35$ & 20 & 27.4 & & & & \\
\hline & Middle & $36-50$ & 28 & 38.4 & 44.7 & 13.4 & 22 & 87 \\
\hline & Old & $>50$ & 25 & 34.2 & & & & \\
\hline \multirow{7}{*}{$\begin{array}{l}\text { Educational } \\
\text { qualification } \\
\text { (Schooling } \\
\text { years) }\end{array}$} & Illiterate & 0 & 5 & 6.8 & & & & \\
\hline & Sign & 0.5 & 17 & 23.3 & & & & \\
\hline & Primary & $1-5$ & 11 & 15.1 & & & & \\
\hline & Secondary & $6-10$ & 30 & 41.1 & 6.47 & 4.7 & 0 & 18 \\
\hline & HSC & $11-12$ & 6 & 8.2 & & & & \\
\hline & Bachelor & $13-16$ & 2 & 2.7 & & & & \\
\hline & Graduate & $17-18$ & 2 & 2.7 & & & & \\
\hline \multirow{3}{*}{$\begin{array}{l}\text { Family size } \\
\text { (No. of family } \\
\text { members) }\end{array}$} & Small & $\leq 4$ & 39 & 53.4 & & & & \\
\hline & Medium & $5-6$ & 19 & 26 & 5.1 & 2.69 & 2 & 20 \\
\hline & Large & $\geq 6$ & 15 & 20.5 & & & & \\
\hline \multirow{3}{*}{$\begin{array}{l}\text { Farming } \\
\text { experience } \\
\text { (Years) }\end{array}$} & Low & $<10$ & 10 & 13.7 & & & & \\
\hline & Medium & $10-20$ & 26 & 35.6 & 24.83 & 14.18 & 2 & 70 \\
\hline & High & $>20$ & 37 & 50.7 & & & & \\
\hline \multirow{5}{*}{$\begin{array}{l}\text { Farm size } \\
\text { (Decimal) }\end{array}$} & Landless & $\leq 4.94$ & 0 & 0 & & & & \\
\hline & Marginal & $4.94-49.43$ & 0 & 0 & & & & \\
\hline & Small & $\begin{array}{l}49.44- \\
247.16\end{array}$ & 39 & 53.4 & 275.06 & 270.86 & 13 & 1350 \\
\hline & Medium & $\begin{array}{l}247.17- \\
741.47\end{array}$ & 19 & 26 & & & & \\
\hline & Large & $>741.47$ & 15 & 20.5 & & & & \\
\hline \multirow[t]{3}{*}{$\begin{array}{l}\text { Cosmopolitanism } \\
\text { (Score) }\end{array}$} & Low & $\leq 5$ & 36 & 49.3 & & & & \\
\hline & Medium & $6-10$ & 36 & 49.3 & 5.2 & 2.43 & 1 & 12 \\
\hline & High & $>10$ & 1 & 1.4 & & & & \\
\hline \multirow{3}{*}{$\begin{array}{l}\text { Organizational } \\
\text { participation } \\
\text { (Score) }\end{array}$} & Low & $\leq 7$ & 62 & 84.9 & & & & \\
\hline & Medium & $8-14$ & 6 & 8.2 & 4.19 & 7.4 & 0 & 40 \\
\hline & High & $>14$ & 5 & 6.8 & & & & \\
\hline \multirow{3}{*}{$\begin{array}{l}\text { Extension } \\
\text { media contact } \\
\text { (Score) }\end{array}$} & Low & $\leq 8$ & 29 & 39.7 & & & & \\
\hline & Medium & $9-16$ & 42 & 57.5 & 9.2 & 3.38 & 2 & 18 \\
\hline & High & $>16$ & 2 & 2.7 & & & & \\
\hline
\end{tabular}


Bangladesh Rural Advancement Committee (BRAC), NGOs, Bangladesh Fisheries Research Institute (BFRI), Syngenta, BLUE GOLD, ISPAHANI AGRO LTD, Bangladesh Livestock Research Institute (BLRI), SHAFOL have provided training to the respondents. Among these trainings, maximum agricultural trainings were provided by DAE, after that, BRAC and NGOs play a vital role in this aspect.

\section{Suitable and cost effective weed control method according to farmers' opinion}

All the respondents (100\%) considered the physical method of weeding as the best control measure of weeds (Table 02) as they were concerned about soil health because of chemical application results in degradation of soil health. According to interviews with the respondents, it was found that $100 \%$ of farmers consider the chemical method of weeding (Table 02) as a cost effective method. However, data were not collected on the actual cost incurred for chemical control measures for weeding in the farmers' field. They explained that for physical control they had to appoint labor that was costly for them. As per the opinion provided by the farmers during the interviews, they considered chemical control as the cheapest method of weeding, as that method required very less number of laborer deployments for weed control.

Singh et al., (1991) reported that yield of rice in weed-free condition was $2.77 \mathrm{t} \mathrm{ha}^{-1}$ whereas in weedy condition yield was $1.82 \mathrm{t}$ ha- 1 and yield loss occurred was $34.3 \%$.In green gram, yield in weed-free and weedy conditions were $1.12 \mathrm{t}$ $\mathrm{ha}^{-1}$ and $0.58 \mathrm{t} \mathrm{ha}^{-1}$ respectively where yield loss was $48.2 \%$ (Sandhu et al., 1993).

\section{Relationship among weeding cost, production cost, and income}

Usually, farmers do not have proper knowledge about these aspects. The labor given by farmers in their field during crop production is generally not considered by them in monetary terms. They usually do not include their labor in the cost of production. However, during the interview with the respondents, information has been recorded about their weeding cost, production cost and seasonal income. From the bar diagram, it is clearly understood that farmers have to spend about $40 \%$ of the total production cost on weeding which results in a reduction of their net income (Fig. 6).If the weeding cost is reduced, it will reduce the production cost which will ultimately result in an increase of their income from crops.

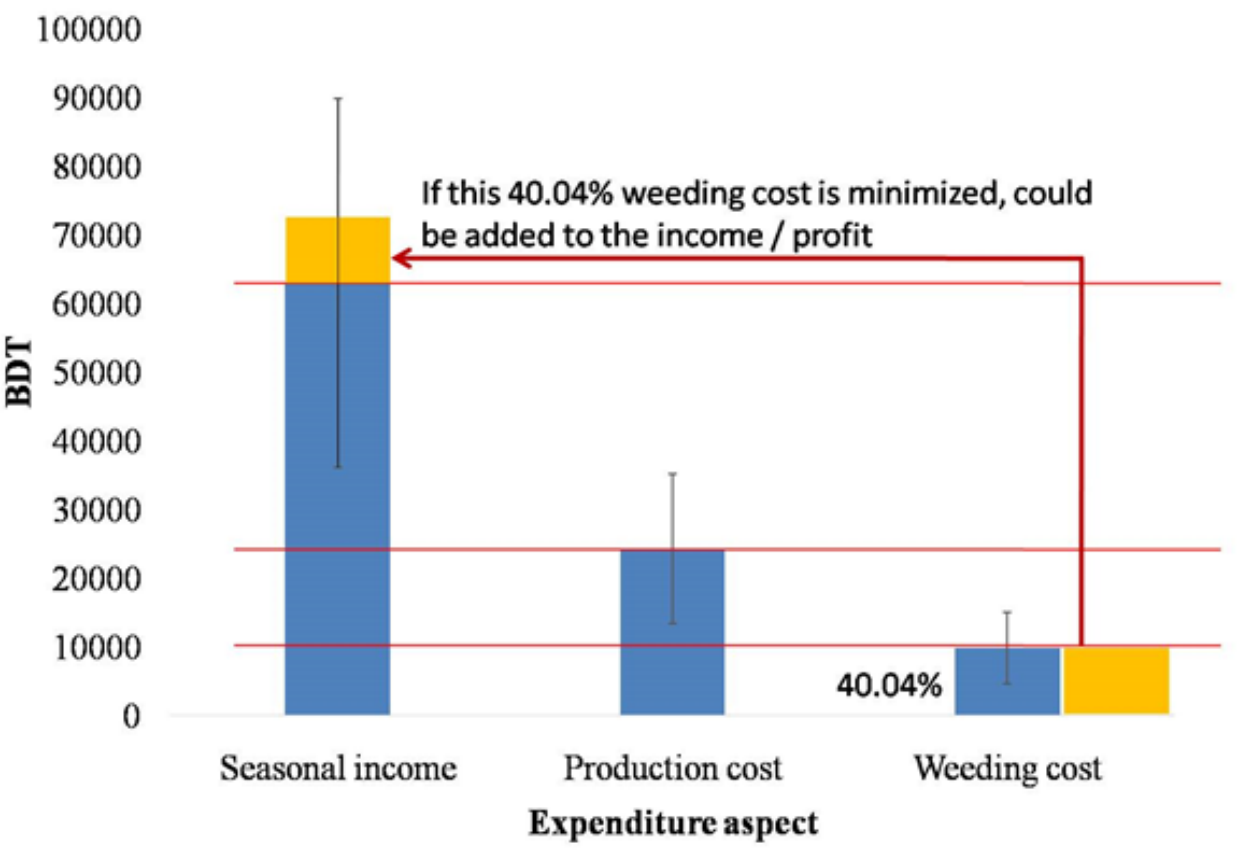

Figure 06. Graphical presentation of weeding cost, production cost and seasonal income. 
Yadav et al., (1995) reported that yield of mustard in weed-free and weedy condition was 1.85 tha $^{-1}$ and 1.23 tha $^{-1}$ respectively where yield loss occurred was $33.5 \%$.In cauliflower, Porwal and Singh (1993) reported $8.08 \mathrm{t} \mathrm{ha}^{-1}$ yield in weed free condition, $4.16 \mathrm{t} \mathrm{ha}^{-1}$ yield in weedy condition and yield loss occurred was $48.5 \%$. Singh et al., (1993) reported that yield of okra in weed-free condition was $10.68 \mathrm{tha}^{-1}$ whereas in weedy condition, yield was $1.09 \mathrm{t} \mathrm{ha}^{-1}$ and yield loss occurred was $89.8 \%$.In potato, yield in weed free and weedy condition was $18.8 \mathrm{t}$ $\mathrm{ha}^{-1}$ and $13.5 \mathrm{t} \mathrm{ha}^{-1}$ respectively, where yield loss was $28.2 \%$ (Singh and Lal, 1994).Clayton et al. (2002) reported that knowledge of the critical period of weed control can also allow towards reduced herbicide use, if farmers can manage weeds with one well-timed herbicide application instead of two. Thus, it is undoubtedly proved that weeding operation during critical weed control period is important for protecting the environment and for gaining economic benefit.

\section{CONCLUSIONS}

There were different weeds associated with Rabi crops with different severity indexes in the study area. Cynodon dactylon was the most dominant weed species in this study area, followed by Cyperus rotundus and Cucumis melo var. agrestis. Among the 17 crops, weeding was done by the farmers within the critical period of weed crop competition in five crops (rice, potato, bitter gourd, spinach and country bean) only. About half (45.2\%) of the farmers had no or low education, with small farm size (53.4\%), low extension media contact (39.7\%) and no training $(63.01 \%)$ and such might be resulting the ineffective cost incurring weed control activities in the study area. Farmers practiced the physical method to control the weeds. According to farmer statement, chemical control method is the cost-effective method of weed control. Sometimes farmers conduct a number of unnecessary weeding sessions before or after a suitable weeding regime. For this reason, they have to spend extra money which increases the production cost. If the weeding cost is reduced, it will reduce the production cost which will ultimately result in an increase in their income from crops. Further research should also be conducted in other parts of Bangladesh to identify associated weeds of Rabi crops more precisely to impose control measures during the critical weeding regime to reduce incurred weeding costs.

\section{CONFLICT OF INTEREST}

There is none competing for the interests regarding the submitted manuscript, and the conducted research, except the authors mentioned in the author list.

\section{ACKNOWLEDGEMENT}

The authors express their heartiest gratitude and indebtedness to all the respondents of the study area who have cooperated with the authors by providing valuable information during data collection.

\section{REFERENCES}

Acharya, S.S., \& Bhattacharya, S.P. (2013). Comparative efficacy ofPyrazosulfuron Ethyl and Bentazon with Acetamides for weed control in transplanted Boro rice (Oryza sativa L.) in the lower Gangetic plain zone of West Bengal, India. International Journal of Bio-Resource \& Stress Management. 4(4), pp. 506-509.

BBS(BangladeshBureau ofStatistics).(2018).Yearbook ofagricultural statistics ofBangladesh-2017, Statistics and Informatics Division (SID), Ministry of Planning, Government of the People's Republic of Bangladesh, Dhaka.

Chowdhury, M.A.H. \& Hassan, M.S. (2013). Handbook of agricultural technology. Bangladesh Agricultural Research Council (BARC), Farmgate, Dhaka, Bangladesh.http://www. barcapps.gov.bd/documents/books/Hand\%20Book\%20of\%20Agricultural\%20Technology. pdf30.10.2019. 
Clayton, G.W., Harker, K.N., O’Donovan, J.T., Baig, M.N. \& Kidnie, M.J. (2002). Glyphosate timing and tillage system effects on glyphosate-resistant canola (Brassica napus). Weed Technology. 16:124-130.

Dangwal, L.R., Singh, A., Singh, T., Sharma, A. \& Sharma, C. (2010).Common weedsof Rabi (winter) crops of TehsilNowshera, District Rajouri (Jammu and Kashmir), India.Journal of American Science. 16(1), pp. 39-45.

Gupta, O.P. (1998). Modern weed management with special reference to agriculture in the tropics and subtropics. New Delhi: Mrs. Sarashwati. pp. 1-24.

Gupta, O.P., Chaudhary, G.R., Bansal, R.G. \& Didwania, G.D. (1979). Evaluation of response of potato to chemical control of early weed infestation. Institute of Agriculture and Animal Science (IAAS) Journal. 1(4),pp. 139-143.

Huda, M., Begum, M., Rahman, M.M., \& Akter, F. (2017). Weed composition study on wheat and boro rice in research and farmers' fields. Journal of Bangladesh Agricultural University. 15(2), pp. 148-157.

Islam, F., Salam, M.A. \& Kader, M.A. (2018). Effect of different weed management techniques on the performance of wheat cultivars. Journal of Bangladesh Agricultural University. 16(3), pp. 437-443.

Jordan, N. (1996). Weed prevention: Priority research for alternative weed management. Journal of Production Agriculture. 9, pp. 485-490.

Knezevic, S.Z., Evans, S.P., Blankenship, E.E., Van Acker, R.C. \& Lindquist, J.L. (2002). Critical period for weed control: the concept and data analysis. Weed science, 773-786.

Mukhopadhayay, S.K. (1992). Emerging problems and advances in weed management. Ind. Sci. Cong., 79th session, Calcutta, India, pp. 1-16.

Norris, R.F. (2007). Weed fecundity: current status and future needs. Crop Protection. 26 (3),pp. $182-188$.

Ontario Crop IPM. (2020). Critical Weed-free Period, Ministry of Agriculture, Food and Rural Affairs, Canada. http://www.omafra.gov.on.ca/IPM/english/weeds-herbicides/critical-weedfree.html 24.10.2020.

Porwal, M.K. \& Singh, M.M. (1993). Efficacy of herbicides for weed control in cauliflower.Indian Journalof Weed Science. 23(1\&2), pp. 55-60.

Rethinam P., Selvaranga R.G., Sankaran N., Sanskaran S. \& Morachan Y.B. (1976). Studies on crop weed competition and chemical weed control in groundnut. Madras Agricultural Journal. 63(8-10), pp. 454-457.

Sandhu K.S., Sandhu B.S. \& Bhatia R.K. (1993). Studies on weed control in mungbean (Vignaradiata). Indian Journalof Weed Science. 25 (1\&2): 61-65.

Singh C. M., Singh S. D. and Suresh, K. (1993). Study on weed management in okra. Indian Journalof Weed Science, 25 (1\&2), pp. 92-95. 
Singh, C., Katti, G.V. \& Tiwari, O.P. (1971). Effect of weed competition on rain fed cotton in black cotton soils. Indian Journalof Agronomy. 16, pp. 137-138

Singh, G., Pant, P.C. \&Bhan, V.M. (1980).Studies on the critical period of weedcontrol in spring planted sugarcane.Indian Journal of Weed Science.12(2), pp. 120-124.

Singh, G., Ram, I.C. \& Singh, D. (1991). Crop-weed competition studies in greengram and blackgram.Tropical Pest Management. 37(2), pp. 144-148.

Singh, K. \& Lal, S.S. (1994). Herbicidal weed control efficiency in potato under N.E. hill conditions. Indian Journal of Agronomy. 39(2), pp. 336-339.

Singh, M. (1978). Crop competition studies in mungbeans. Indian Journal of Agronomy. 23(4), pp. 377-378.

Walia, U.S. \& Walia, S.S.(2015).Crop Management. Rajasthan 342001, India: Scientific Publishers. p. 74.

Weed Management :: Critical Stages of Weed Competition. (2019). http://agritech.tnau.ac.in/ agriculture/agri_weemgt_criticalperiodofweedcompetition.html 30.10.2019.

Yadav, R.P., Shrivastava, U.K. \& Yadav, K.S. (1995). Yield and economic analysis of weed control practices in Indian mustard. Indian Journal of Agronomy.40 (1),pp. 122-124. 